\title{
Magnetic ordering and quantum statistical effects in strongly repulsive Fermi-Fermi and Bose-Fermi mixtures
}

\author{
X.-W. Guan, ${ }^{1}$ M. T. Batchelor, ${ }^{1,2}$ and J.-Y. Lee ${ }^{1}$ \\ ${ }^{1}$ Department of Theoretical Physics, Research School of Physical Sciences and Engineering, Australian National University, \\ Canberra ACT 0200, Australia \\ ${ }^{2}$ Mathematical Sciences Institute, Australian National University, Canberra ACT 0200, Australia
}

(Received 17 June 2008; published 15 August 2008)

\begin{abstract}
We investigate magnetic properties and statistical effects in one-dimensional (1D) strongly repulsive twocomponent fermions and in a 1D mixture of strongly repulsive polarized fermions and bosons. Universality in the characteristics of phase transitions, magnetization, and susceptibility in the presence of an external magnetic field $H$ are analyzed from the exact thermodynamic Bethe ansatz solution. We show explicitly that polarized fermions with a repulsive interaction have antiferromagnetic behavior at zero temperature. A universality class of linear field-dependent magnetization persists for weak and finite strong interaction. The system is fully polarized when the external field exceeds the critical value $H_{c}^{F} \approx \frac{8}{\gamma} E_{F}$, where $E_{F}$ is the Fermi energy and $\gamma$ is the dimensionless interaction strength. In contrast, the mixture of polarized fermions and bosons in an external field exhibits square-root field-dependent magnetization in the vicinities of $H=0$ and the critical value $H=H_{c}^{M} \approx \frac{16}{\gamma} E_{F}$. We find that a pure boson phase occurs in the absence of the external field, fully polarized fermions and bosons coexist for $0<H<H_{c}^{M}$, and a fully polarized fermion phase occurs for $H \geqslant H_{c}^{M}$. This phase diagram for the Bose-Fermi mixture is reminiscent of weakly attractive fermions with population imbalance, where the interacting fermions with opposite spins form singlet pairs.
\end{abstract}

DOI: 10.1103/PhysRevA.78.023621

PACS number(s): 03.75.Ss, 05.30.Fk, 71.10.Pm

\section{INTRODUCTION}

The recent success in experimentally realizing degenerate quantum gases in low dimensions [1-5] has revived interest in one-dimensional (1D) integrable models of interacting fermions and bosons [6-13]. The 1D atomic gases with internal degrees of freedom are tunable interacting many-body systems featuring novel strong correlation effects and subtle quantum phase transitions. Exotic quantum phases in 1D two-component attractive fermions have received considerable interest [14-31] due to the experimental observation of fermionic superfluidity and phase transitions [32-34]. For certain regimes, the two-component Fermi gas with population imbalance can be viewed as a strongly interacting BoseFermi mixture $[35,36]$. However, subtle differences between the bosonic dimer and pure bosons have been observed experimentally $[37,38]$. For repulsive interaction, the twocomponent Fermi gas exhibits antiferromagnetic behavior $[15-18,39,40]$. In contrast to the two-component Fermi gas, the 1D spinor Bose gases [10,41-44] possess novel ferromagnetic properties and collective dynamics of spin waves at temperatures below the degenerate temperature. The subtlety in 1D quantum many-body physics [45] is a result of the dynamical interaction between identical particles and their statistics.

On the other hand, the recent success in tuning interspecies Feshbach resonances in Bose-Fermi mixtures of ultracold atoms $[37,38,46]$, such as ${ }^{6} \mathrm{Li}+{ }^{7} \mathrm{Li},{ }^{6} \mathrm{Li}+{ }^{23} \mathrm{Na},{ }^{40} \mathrm{~K}$ $+{ }^{87} \mathrm{Rb}$, and ${ }^{6} \mathrm{Li}+{ }^{87} \mathrm{Rb}$, opens up a new gateway for exploring many-body physics, including superfluids and Mott insulators, spin and charge density waves, phase separation, the BCS-BEC crossover, etc. On the theoretical side, BoseFermi mixtures have been studied through various techniques like the mean-field approximation [47], Luttinger liq- uid formalism [48,49], quantum Monte Carlo [50], bosonization techniques [51], exact solutions using the Bethe ansatz [52-54], and other methods [55]. However, the role of quantum fluctuations is enhanced in 1D compared to the three-dimensional case to the extent that traditional meanfield theories fail for strong interaction in 1D. The exact Bethe ansatz solution of 1D many-body systems provides more reliable physics than the mean-field theory.

In this paper, we investigate external field-dependent magnetic properties and statistical effects of 1D twocomponent fermions with repulsion and a 1D mixture of polarized fermions and bosons by means of their exact thermodynamic Bethe ansatz solution. The antiferromagnetic ground-state properties and critical behavior of twocomponent repulsive fermions are studied in detail. The universality class of linear field-dependent behavior of magnetization is predicted for weak and finitely strong coupling regimes. However, in the Tonks-Girardeau limit, a van Hove singularity in the susceptibility occurs as the external field approaches a critical field. In this limit, the system becomes a system with free spins. However, the existence of bosons in the Bose-Fermi mixture destroys the antiferromagnetic ordering in the two-component interacting fermions. In the absence of the external field, the state of pure bosons is among the ground states. When the external field is turned on, a second-order phase transition from a pure boson phase into a mixed boson-fermion phase occurs. A fully polarized Fermi liquid occurs when the external field exceeds a critical value. We calculate the explicit details of these transitions.

This paper is set out as follows. In Sec. II, we recall the Bethe ansatz solution of the 1D integrable model of mixed bosons and fermions. In Sec. III, we study the antiferromagnetic behavior of $1 \mathrm{D}$ strongly repulsive interacting fermions with polarization. The phase diagram of the model is pre- 
sented. The quantum phase transitions and magnetic properties of the Bose-Fermi mixture are studied in Sec. IV. Section $\mathrm{V}$ is devoted to concluding remarks and a brief discussion.

\section{HAMILTONIAN}

We consider a $\delta$-function interacting system of $N$ bosons and fermions with equal mass $m$ and internal degrees of freedom, or a mixture of two-component fermions and spinless bosons, constrained by periodic boundary conditions to a line of length $L$ in an external magnetic field $H$. The Hamiltonian $[56,57]$ is

$$
H=-\frac{\hbar^{2}}{2 m} \sum_{i=1}^{N} \frac{\partial^{2}}{\partial x_{i}^{2}}+g_{1 \mathrm{D}} \sum_{1 \leqslant i<j \leqslant N} \delta\left(x_{i}-x_{j}\right)-\frac{1}{2} H\left(M_{1}-M_{2}\right) .
$$

The quantum numbers $M_{1}$ and $M_{2}$ are the numbers of fermions with spin up and spin down, respectively. Under exchange of spatial and internal spin coordinates between two particles the wave functions of the Hamiltonian (1) are symmetric for bosons or for fermions with opposite hyperfine states and antisymmetric for fermions with the same hyperfine states.

For maintaining the integrability of the model, we consider the same mass for bosons and fermions and the same interaction strength $g_{1 \mathrm{D}}$ between bosons, between bosons and fermions, and between fermions with opposite hyperfine states. The interaction is attractive for $g_{1 \mathrm{D}}<0$ and repulsive for $g_{1 \mathrm{D}}>0$. There is no $\delta$-type interaction between fermions with the same hyperfine states. Although these conditions appear somewhat restrictive, the model still captures the essential physics [53] relevant to the current experimental observations $[35,37,38]$. The interspecies interaction can be tuned from strongly attractive $\left(g_{1 \mathrm{D}} \rightarrow-\infty\right)$ to strongly repulsive $\left(g_{1 \mathrm{D}} \rightarrow+\infty\right)$ via Feshbach resonances and optical confinement. The coupling constant $g_{1 \mathrm{D}}$ can be written in terms of the scattering strength $c=2 / a_{1 \mathrm{D}}$ as $g_{1 \mathrm{D}}=\hbar^{2} c / m$. In principle, an effective 1D scattering length $a_{1 \mathrm{D}}$ can be expressed through the 3D scattering length for the bosons and fermions confined in a 1D geometry. We use the dimensionless coupling constant $\gamma=m g_{1 \mathrm{D}} /\left(\hbar^{2} n\right)$ for physical analysis. Here $n$ $=N / L$ is the linear density.

This model was solved in 1963 by Lieb and Liniger for the special case of spinless bosons [58]. Experimental realization of 1D interacting Bose gases [1-4] has stimulated further interest in various integrable models, including interacting two-component fermions solved by Yang and Gaudin $[59,60]$, the Bose-Fermi mixture solved by Lai and Yang [57], and multicomponent bosons and fermions solved by Sutherland [56].

The Bethe ansatz equations for the Bose-Fermi mixture (1) with an irreducible representation $\left[2+M_{b}, 2^{M_{2}-1}, 1^{M_{1}-M_{2}}\right]$ are [57]

$$
\exp \left(\mathrm{i} k_{j} L\right)=\prod_{\alpha=1}^{M} \frac{k_{j}-\lambda_{\alpha}+\mathrm{i} c^{\prime}}{k_{j}-\lambda_{\alpha}-\mathrm{i} c^{\prime}},
$$

$$
\begin{gathered}
\prod_{j=1}^{N} \frac{\lambda_{\alpha}-k_{j}+\mathrm{i} c^{\prime}}{\lambda_{\alpha}-k_{j}-\mathrm{i} c^{\prime}}=-\prod_{\beta=1}^{M} \frac{\lambda_{\alpha}-\lambda_{\beta}+\mathrm{i} c}{\lambda_{\alpha}-\lambda_{\beta}-\mathrm{i} c} \prod_{b=1}^{M_{b}} \frac{\lambda_{\alpha}-A_{b}-\mathrm{i} c^{\prime}}{\lambda_{\alpha}-A_{b}+\mathrm{i} c^{\prime}}, \\
\prod_{k=1}^{M} \frac{A_{b}-\lambda_{k}-\mathrm{i} c^{\prime}}{A_{b}-\lambda_{k}+\mathrm{i} c^{\prime}}=1 .
\end{gathered}
$$

We define $M=M_{2}+M_{b}$, where $M_{b}$ is the number of bosons. In these equations $k_{j}$, with $j=1, \ldots, N$, are the quasimomenta of the particles and $\lambda_{\alpha}$, with $\alpha=1, \ldots, M$, are parameters for the fermions with spin down and bosons. $A_{b}$ with $b$ $=1, \ldots, M_{b}$ are the parameters for the bosons. In the Bethe ansatz process [57], the conjugate representation $\left[M_{1}, M_{2}, 1^{2+M_{b}}\right]$ was used to solve matrix eigenvalue equations associated with the irreducible representation [2 $\left.+M_{b}, 2^{M_{2}-1}, 1^{M_{1}-M_{2}}\right]$ following Sutherland's paper [56].

The Bethe ansatz solution of the Bose-Fermi mixture was considered with renewed interest [52-54] due to the experimental observation of new quantum phases [37,38]. The ground-state properties, correlation functions, and harmonic trapping for the mixture of fully polarized fermions and bosons were studied in Ref. [53]. The model of arbitrarily polarized fermions and spinless bosons was investigated in the weak coupling regime [54]. The Bethe ansatz equations (2) also contain those for two-component fermions [59] when $M_{b}=0$. However, the magnetic properties and phase transitions for two-component fermions in the repulsive regime and the mixture of polarized fermions and bosons have not been comprehensively investigated. We turn now to the investigation of their magnetic properties and phase transitions in the repulsive regime.

\section{TWO-COMPONENT FERMIONS}

It is well known that the ground state for $1 \mathrm{D}$ interacting fermions is antiferromagnetic. This is proved to be the case by the Lieb-Mattis theorem [61]. They showed that for a system with total spin $S^{\prime}>S$, the lowest energy belonging to that system is also greater, $E\left(S^{\prime}\right)>E(S)$. Hence when there is no external field, the system is not polarized. Another way of showing this is from the Pauli exclusion principle. When there is no interaction at all, the ground state can only have one pair of fermions (spin up and spin down) having a particular pseudomomentum $k$. When a repulsive interaction $\gamma$ is "switched" on, the pair will split so that no two fermions can have the same value for $k$. However, the sequence of spin-up and spin-down fermions is still unaltered, which shows that they remain antiferromagnetic even when $\gamma>0$. The magnetization of the two-component fermion system in the weak coupling limit, $\gamma \ll 1$, can be directly obtained from the ground-state energy derived from the discrete Bethe ansatz equations [17]

$$
\frac{E}{L} \approx \frac{c}{2} n^{2}\left(1-P^{2}\right)+\frac{\pi^{2}}{12} n^{3}+\frac{\pi^{2}}{4} n^{3} P^{2},
$$

where in a notation the polarization $P=\left(N_{\uparrow}-N_{\downarrow}\right) / N$. Defining the magnetization per unit length as $m^{z}=n P / 2$, the magnetization has a linear field-dependent form given by 


$$
H \approx 2 n\left(\pi^{2}-2 \gamma\right) m^{z} .
$$

When the external field exceeds the critical value $H_{c}^{F}$ $\approx n^{2}\left(\pi^{2}-2 \gamma\right)$ a fully polarized phase occurs. The magnetic susceptibility is $\chi \approx 1 / 2 n\left(\pi^{2}-2 \gamma\right)$ for finite $H$. However, from field theory, $\chi \approx 1 /\left(2 \pi v_{s}\right)$ at $H=0$. Here the spin velocity is $v_{s} \approx n \pi\left(1-\gamma / \pi^{2}\right)[15,45,62]$.

We turn now to the magnetic properties of the $1 \mathrm{D}$ exactly solved model of two-component fermions with arbitrary polarization in the strong coupling regime $\gamma \gg 1$. In the thermodynamic limit, $L, N \rightarrow \infty$ with $N / L$ finite, the Bethe ansatz equations can be written in terms of the spin and charge densities in the form $[59,60]$

$$
\begin{aligned}
\rho(k)+\rho^{h}(k)=\frac{1}{2 \pi} & +\frac{1}{2 \pi} \int_{-B}^{B} \frac{c \sigma(\lambda)}{c^{2} / 4+(k-\lambda)^{2}} d \lambda, \\
\sigma(\lambda)+\sigma^{h}(\lambda)= & \frac{1}{2 \pi} \int_{-Q}^{Q} \frac{c \rho(k)}{c^{2} / 4+(\lambda-k)^{2}} d k \\
& -\frac{1}{2 \pi} \int_{-B}^{B} \frac{2 c \sigma(\lambda)}{c^{2}+\left(\lambda-\lambda^{\prime}\right)^{2}} d \lambda^{\prime} .
\end{aligned}
$$

The integration limits $Q$ and $B$ are determined by the total number of particles $N / L=\int_{-Q}^{Q} \rho(k) d k$ and the number of spindown fermions $M_{2} / L=\int_{-B}^{B} \sigma(\lambda) d \lambda$. We denote $\rho(k)$ and $\rho^{h}(k)$ $\left[\sigma(k)\right.$ and $\left.\sigma^{h}(k)\right]$ as the densities of particles and holes for charge (spin) degrees of freedom, respectively. For the ground state $\rho^{h}(k)=\sigma^{h}(\lambda)=0$, i.e., there are no holes for both charge and spin rapidities.

The integral equations (5) and (6) provide the ground state and excited states at zero temperature. At finite temperatures the equilibrium states are determined by the thermodynamic Bethe ansatz (TBA) equations $[63,64]$. The TBA equations in the zero temperature limit lead to the so-called dressed energy equations which describe the band fillings in the Fermi seas driven by external fields. Consequently, from the band filling configurations we can analyze the phase transitions and derive the ground-state energy and magnetic properties of the two-component repulsive fermion model with an external magnetic field. In terms of the dressed energies $\epsilon(k):=T \ln \left[\rho^{h}(k) / \rho(k)\right]$ for the charge and $\phi(\lambda)$ $:=T \ln \left[\sigma^{h}(\lambda) / \sigma(\lambda)\right]$ for the spin degrees of freedom, these equations are

$$
\begin{gathered}
\epsilon(k)=k^{2}-\mu-\frac{1}{2} H+\frac{1}{2 \pi} \int_{-B}^{B} \frac{c \phi^{-}(\lambda)}{c^{2} / 4+(k-\lambda)^{2}} d \lambda, \\
\phi(\lambda)=g_{1}(\lambda)-\frac{1}{2 \pi} \int_{-B}^{B} \frac{2 c \phi^{-}\left(\lambda^{\prime}\right)}{c^{2}+\left(\lambda-\lambda^{\prime}\right)^{2}} d \lambda^{\prime},
\end{gathered}
$$

where the driving term is given by

$$
g_{1}(\lambda)=H+\frac{1}{2 \pi} \int_{-Q}^{Q} \frac{c \epsilon^{-}(k)}{c^{2} / 4+(\lambda-k)^{2}} d k .
$$

The negative part of the dressed energy, $\epsilon(k) \leqslant 0$ for $|k|$ $\leqslant Q$, or $\phi(\lambda) \leqslant 0$ for $|\lambda| \leqslant B$, corresponds to occupied states, with the positive part of $\epsilon(k)$ and $\phi(\lambda)$ corresponding to un- occupied states. We clearly see from the TBA equations (7) and (8) that the spin interaction is antiferromagnetic with an effective spin-spin exchange interaction depending on the energy of the system. For $H=0$, the driving term gives rise to an asymptotic condition $\phi(\infty)=0$ below the $\lambda$ axis. This leads to a maximum $\phi^{-}(\lambda)$ which gives the lowest energy state of the system. For strong coupling $\gamma \gg 1$, the driving terms in the second TBA equation $(8)$ become $g_{1}(\lambda) \approx H$ $-c P_{0} /\left(c^{2} / 4+\lambda^{2}\right)$ where we ignore $O\left(1 / c^{2}\right)$ contributions. Here $P_{0}=-\frac{1}{2 \pi} \int_{-Q}^{Q} \epsilon^{-}(k) d k$ is the pressure. The driving term $g_{1}$ clearly indicates that the form of Eq. (8) indicates an effective antiferromagnetic spin-spin exchange interaction with an effective coupling constant $J=-2 P_{0} / c<0$ in contrast to the effective ferromagnetic coupling in spinor Bose gas [43,44], for which $J=2 P_{0} / c>0$. For the ground state we find that $\phi_{0}(\lambda)=-\frac{\pi P_{0}}{c \cosh (\pi \lambda / c)}$. On solving the TBA equations (7) and (8), the leading behavior for the energy per unit length and the chemical potential is given by

$$
\begin{gathered}
F(0) / L=\frac{1}{3} n^{3} \pi^{2}\left(1-\frac{4 \ln 2}{\gamma}\right), \\
\mu=n^{2} \pi^{2}\left(1-\frac{16 \ln 2}{3 \gamma}\right),
\end{gathered}
$$

which coincide with previous results [15].

\section{A. Finite external field $\boldsymbol{H}$}

When the external field is applied, the spin-up states are energetically favoured due to Zeeman splitting. As the magnetic field increases, the Fermi sea of the spin sector $\phi(\lambda)$ is raised. For small field, $H \ll 1$, the second TBA equation (8) becomes

$$
\phi(\lambda)=\frac{1}{2} H-\frac{2 \pi P_{0}}{2 c \cosh (\pi \lambda / c)}+\int_{-\infty}^{\infty} G\left(\lambda-\lambda^{\prime}\right) \phi^{+}\left(\lambda^{\prime}\right) d \lambda^{\prime},
$$

with the function

$$
G(\lambda)=\frac{1}{2 \pi} \int_{-\infty}^{\infty} \frac{1}{1+e^{|\omega c|}} e^{-i \omega \lambda} d \omega .
$$

Making a change of variables $\lambda \rightarrow \lambda+B$ and defining $y(\lambda)$ $\equiv \phi(\lambda+B)$, Eq. (12) becomes

$$
\begin{aligned}
y(\lambda)= & \frac{1}{2} H-\frac{2 \pi P}{2 c \cosh [\pi(\lambda+B) / c]}+\int_{0}^{\infty} G(\lambda-k) y(k) d k \\
& +\int_{0}^{\infty} G(\lambda+2 B+k) y(k) d k .
\end{aligned}
$$

Observing that $G(\lambda+2 B+k) \rightarrow e^{-2 \pi B / c}$ for $B \gg 1$, we can take the expansion $y=y_{1}+y_{2}+\cdots$ with respect to the order of $e^{-2 \pi m B / c}$, where $m=1,2, \ldots$ It follows that 


$$
\begin{aligned}
y_{1}(\lambda) & +y_{2}(\lambda)+\cdots \\
= & \frac{1}{2} H-2 \pi P_{0} c^{-1} e^{-\pi \lambda / c} e^{-\pi B / c} \\
& +\int_{0}^{\infty} G(\lambda+2 B+k)\left[y_{1}(k)+y_{2}(k)+\cdots\right] d k \\
& +\int_{0}^{\infty} G(\lambda-k)\left[y_{1}(k)+y_{2}(k)+\cdots\right] d k .
\end{aligned}
$$

Collecting terms of the same order then gives

$$
\begin{aligned}
& y_{1}(\lambda)=\frac{1}{2} H-2 \pi P_{0} c^{-1} e^{-\pi \lambda / c} e^{-\pi B / c}+\int_{0}^{\infty} G(\lambda-k) y_{1}(k) d k, \\
& y_{2}(\lambda)=\int_{0}^{\infty} G(\lambda+2 B+k) y_{1}(k) d k+\int_{0}^{\infty} G(\lambda-k) y_{2}(k) d k,
\end{aligned}
$$

and so on. To a sufficient approximation, we only need to consider the equation for $y_{1}(\lambda)$. In considering the leading contribution in powers of the exponential $G(\lambda+2 B+k)$ $\rightarrow e^{-2 \pi B / c}$ for $B \gg 1$, Eq. (12) reduces to the standard WienerHopf equation (for the Wiener-Hopf technique, see, e.g., [64-68]). After tedious calculation we obtain the free energy, pressure, and chemical potential as

$$
\begin{aligned}
& F(H) \approx \frac{1}{3} \pi^{2} n^{3}\left(1-\frac{4 \ln 2}{\gamma}\right)-\frac{3 H^{2} \gamma}{8 \pi^{4} n}\left(1+\frac{12 \ln 2}{\gamma}\right), \\
& P(H) \approx \frac{2}{3} \pi^{2} n^{3}\left(1-\frac{6 \ln 2}{\gamma}\right)+\frac{9 H^{2} \gamma}{8 \pi^{4} n}\left(1+\frac{104 \ln 2}{9 \gamma}\right), \\
& \mu(H) \approx \pi^{2} n^{2}\left(1-\frac{16 \ln 2}{3 \gamma}\right)+\frac{3 H^{2} \gamma}{4 \pi^{4} n^{2}}\left(1+\frac{34 \ln 2}{3 \gamma}\right) .
\end{aligned}
$$

Moreover, the magnetization $m^{z}(H)$ and susceptibility $\chi(H)$ follow in the small field regime using the formula $m^{z}=$ $-\partial F(H) / \partial H$ and $\chi(H)=\partial m^{z} / \partial H$. Hence

$$
\begin{aligned}
& m^{z}(H) \approx \frac{3 H \gamma}{4 \pi^{4} n^{2}}\left(1+\frac{12 \ln 2}{\gamma}\right), \\
& \chi(H) \approx \frac{3 \gamma}{4 \pi^{4} n^{2}}\left(1+\frac{12 \ln 2}{\gamma}\right) .
\end{aligned}
$$

In the small field regime, the higher-order logarithmic correction to the susceptibility can be calculated in the same fashion by solving to next order in the Wiener-Hopf equations. Thus finite logarithmic singularities are expected in the susceptibility $[64,66]$. The linear field-dependent behavior of the magnetization is maintained for finitely strong and weak repulsion. However, for the Tonks-Girardeau limit $\gamma \rightarrow \infty$, caution should be paid to the critical behavior of the polarized fermions. The spin velocity is proportional to the inverse of the susceptibility. Therefore, in this limit the spin velocity $v_{s}$ tends to zero such that the spin propagation is almost frozen. As a consequence the statistical effect is fully suppressed and the Tonks-Girardeau Fermi gas behaves like free spins. Therefore, we expect an infinitely divergent susceptibility to occur for the system in the limit $\gamma \rightarrow \infty$. In the next part, we shall search for evidence of the paramagnetic behavior in the Tonks-Girardeau Fermi gas with population imbalance.

If the external magnetic field is sufficiently strong, the system can be fully polarized. From the TBA equations (7) and (8), we find that if the field $H \geqslant H_{c}^{F}=4 P_{0} / c$, then $\phi(0)$ $\geqslant 0$. The critical value $H_{c}^{F}$ indicates a phase transition from a partially polarized ferromagnetic phase to a fully polarized ferromagnetic phase. For the limit $H \rightarrow H_{c}^{F}$, the system is almost fully polarized, corresponding to a very small Fermi sea for the spin sector of the TBA equation. The critical behavior may be directly derived from the TBA equations (7) and (8) or the Bethe ansatz equations (5) and (6). In the vicinity of $H_{c}^{F}$, we can approximate the integral for the spin part by taking the area under the curve as a rectangle as usually done in spin chains and ladders [67]. In this way

$$
\phi(\lambda) \approx H-\frac{P_{0} c}{c^{2} / 4+\lambda^{2}}-\frac{2 B c \phi^{-}(0)}{\pi\left(c^{2}+\lambda^{2}\right)},
$$

with $\phi(0) \approx \frac{H-4 P_{0} / c}{1+2 B / \pi c}$. Further, $\phi(B)=0$ provides the value of the integration boundary, with

$$
B=c \sqrt{\frac{4 P_{0} / c-H}{5 H-4 P_{0} / c}} .
$$

On the other hand, from the Bethe ansatz equations (6), we find

$$
B=\frac{1}{4} c \pi(1-\sqrt{1-2 \alpha})
$$

if $\alpha=M / N \ll 1$. Here $M=M_{2}$ is the number of spin-down fermions. Thus we have an explicit relation

$$
\alpha=\frac{4}{\pi} \sqrt{\left(H_{c}^{F}-H\right) /\left(5 H_{c}^{F}-H\right)}
$$

between the number of spin-down fermions and the external field, where $H_{c}^{F}=4 P_{0} / c$.

Substituting the expression (23) into the first TBA equation (7) and also using the conditions $\epsilon(Q)=0$ and $\partial P_{0} / \partial \mu$ $=n$, we obtain the leading terms for the pressure and the free energy

$$
\begin{gathered}
P(H) \approx \frac{2}{3} \pi^{2} n^{3}\left(1-\frac{72}{\gamma} \frac{\left(H_{c}-H\right)^{1 / 2}}{\pi\left(5 H-H_{c}\right)^{1 / 2}}\right), \\
F(H) \approx-\frac{1}{2} n H+\frac{1}{3} \pi^{2} n^{3}-\frac{4 n\left(H_{c}-H\right)^{3 / 2}}{\pi\left(5 H-H_{c}\right)^{1 / 2}} .
\end{gathered}
$$

The ground-state energy 


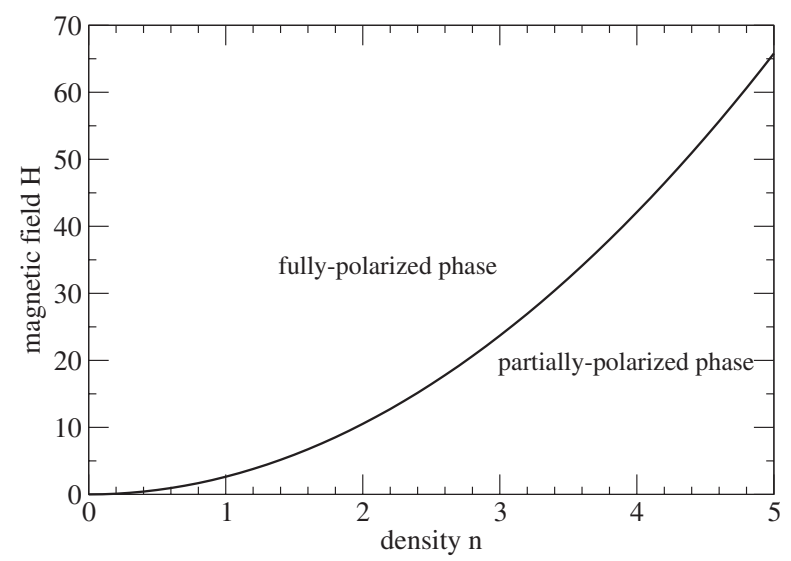

FIG. 1. Phase diagram for 1D polarized fermions with strong coupling $c=10$. All figures are in natural units.

$$
\frac{E}{L} \approx \frac{1}{3} n^{3} \pi^{2}\left(1-\frac{8 \alpha}{\gamma}\right)
$$

can be obtained from the relation $E / L=F(H) / L+H n(1$ $-2 \alpha) / 2$, which is consistent with the BA result [17]. Here recall that $\alpha=M / N$. The critical field $H_{c}^{F} \approx 4 P_{0} / c \approx \frac{8}{\gamma} E_{F}$, where $E_{F}=\frac{1}{3} n^{2} \pi^{2}$ is the Fermi energy. These explicit results indicate that for $\gamma \rightarrow \infty$, the system becomes a paramagnet and statistical interaction is suppressed. It also follows that the magnetization $m^{z}(H)$ and susceptibility $\chi(H)$ in the limit $H \rightarrow H_{c}^{F}$ are given by

$$
\begin{gathered}
m^{z}(H) \approx \frac{n}{2}-\frac{6 n\left(H_{c}-H\right)^{1 / 2}}{\pi\left(5 H-H_{c}\right)^{1 / 2}}-\frac{10 n\left(H_{c}-H\right)^{3 / 2}}{\pi\left(5 H-H_{c}\right)^{3 / 2}}, \\
\chi(H) \approx \frac{3 n}{\pi\left(5 H-H_{c}\right)^{1 / 2}\left(H-H_{c}\right)^{1 / 2}}+\frac{15 n\left(H_{c}-H\right)^{1 / 2}}{\pi\left(5 H-H_{c}\right)^{3 / 2}} .
\end{gathered}
$$

The expression for the susceptibility is seen to be divergent when $H \rightarrow H_{c}^{F}$. A quantum phase transition occurs at the critical value $H_{c}^{F}$ (see the phase diagram in Fig. 1). The squareroot behavior of the magnetization is a consequence of the van Hove singularity in one dimension.

The analytic results for the magnetic properties show that spin-spin interaction for the system of the polarized fermions with strong coupling $\gamma \gg 1$ can be effectively described by the isotropic spin-1/2 Heisenberg chain with a weak antiferromagnetic coupling $J \approx-4 E_{F} / \gamma$. For the isotropic spin-1/2 Heisenberg chain [64], the susceptibility at $H=0$ is given by $\chi \approx 1 / J \pi^{2}$, which coincides with the result (22). Furthermore, we can find the spin velocity at $H=0$,

$$
v_{s} \approx \frac{2 \pi^{3} n}{3 \gamma}\left(1-\frac{12 \ln 2}{\gamma}\right) .
$$

The charge velocity can be calculated from the ground-state energy (10) [17], for example, for $H=0$,

$$
v_{c} \approx v_{F}\left(1-\frac{4 \ln 2}{\gamma}\right),
$$

where the Fermi velocity $v_{F}=\hbar \pi n / m$. The antiferromagnetic spin-spin exchange interaction embedded in 1D interacting polarized fermions may provide insight into understanding the spin segregation in a trapped Fermi gas [69].

\section{B. Summary}

Before ending this section we summarize the critical behavior of the two-component fermion model with repulsive interaction. The dressed energy equations (7) and (8) indicate that the spin-spin interaction in the repulsive Fermi gas is antiferromagnetic. The lowest state with energy (10) corresponds to an equal number of spin-up and spin-down fermions. Dynamical interaction between fermions with opposite spins leads to spin-charge separation with velocities (32) and (33), which can essentially describe the low energy physics determined by the Bethe ansatz equations (5) and (6) [17]. At zero temperature spin wave bound states do not populate the ground state. For very small magnetic field, the linear fielddependent magnetization (21) and finite susceptibility (22) are an important antiferromagnetic signature. The linear field-dependent magnetization (4) and (21) is maintained for weak and finitely strong interaction due to the antiferromagnetic spin-spin interaction. However, logarithmic corrections to the susceptibility may not be negligible as the interaction strength increases. The critical field $H_{c}^{F}$ is proportional to the Fermi energy. If the external field exceeds this critical value, infinite divergence in the susceptibility (31) in the vicinity of $H_{c}^{F}$ is found for the strong coupling limit. In this limit, the spin and charge degrees of freedom are fully separated and the quantum statistics of the particles is suppressed. Our analytic results for the magnetic properties provide accessible critical scalings for two-component repulsive interacting fermions which are difficult to extract via numerical schemes.

\section{BOSE-FERMI MIXTURE}

In this section, we shall use the TBA approach to investigate the ground state and magnetic properties of the 1D Bose-Fermi mixture. We analyze the band filling configurations to solve analytically the dressed energy equations in the presence of the external field in order to obtain magnetic properties. We shall see that under a magnetic field, the system possesses three phases: (I) a pure boson phase; (II) a mixed boson-fermion phase; and (III) a fully polarized fermion phase.

The earliest study of the ground state of the 1D mixture of bosons and polarized fermions dates back to Lai and Yang in 1971 [57] who showed by numerically solving a set of coupled integral equations that the energy of the system is a monotonic decreasing function with respect to the ratio of bosons and the total number of particles in the system. This implied that the ground state is occupied only by bosons. The same signature was found recently by other groups [52-54]. The ground-state energy for the weak coupling regime was given explicitly in Ref. [54]. In addition, study of the quasi- 
momentum distributions [70] showed that the quasimomentum distribution is more compressed when there are no fermions. As the number of fermions increases, the quasimomentum distribution becomes more spread out due to the Fermi pressure.

It is thus natural to ask what the true physical ground state is in the presence of an external magnetic field. What kind of magnetism does the model exhibit? As far as we understand, the magnetic properties of the mixture of bosons and polarized fermions have not been studied yet. It is of interest to know how the addition of bosons influences the antiferromagnetic ground-state properties of the spin- $\frac{1}{2}$ fermionic system. This provides further insight in understanding the signature of the strongly interacting Bose-Fermi mixture and the two-component Fermi-Fermi mixture with population imbalance [35]. Application of an external magnetic field to the polarized fermions causes Zeeman splitting of the spin-up and spin-down fermions into different energy levels. The ground state can only accommodate fermions that are in the lower energy level. Therefore it is expected that when the direction of the magnetic field is along the spin-up $(H>0)$ direction, spin-down fermions can no longer populate the ground state. In such a way, the system (1) naturally reduces to a mixture of a fully polarized Fermi gas and a Bose gas.

The derivation of the TBA equations for the model (1) is standard [71]. Here we present a set of dressed energy equations which is convenient for the analysis of the ground-state properties. These equations are

$$
\begin{aligned}
& \epsilon(k)=k^{2}-\mu-\frac{1}{2} H+\frac{1}{2 \pi} \int_{-B}^{B} \frac{c \phi^{-}(\lambda)}{\frac{c^{2}}{4}+(k-\lambda)^{2}}, \\
& \phi(\lambda)=H+\frac{1}{2 \pi} \int_{-Q}^{Q} \frac{c \epsilon^{-}(k)}{\frac{c^{2}}{4}+(\lambda-k)^{2}}+\frac{1}{2 \pi} \int_{-d}^{d} \frac{c \psi^{-}(\Lambda)}{\frac{c^{2}}{4}+(\lambda-\Lambda)^{2}} \\
& -\frac{1}{2 \pi} \int_{-B}^{B} \frac{2 c \phi^{-}\left(\lambda^{\prime}\right)}{c^{2}+\left(\lambda-\lambda^{\prime}\right)^{2}} \\
& \psi(\Lambda)=-\mu_{b}-\frac{H}{2}+\frac{1}{2 \pi} \int_{-B}^{B} \frac{c \phi^{-}(\lambda)}{\frac{c^{2}}{4}+(\Lambda-\lambda)^{2}} .
\end{aligned}
$$

The chemical potentials $\mu$ and $\mu_{b}$ are for the total number of particles and for bosons. The magnetic field is again denoted by $H$. The dressed energies are defined [71] by $\exp [\epsilon(k) / T]$ $=\rho^{h}(k) / \rho(k), \exp [\phi(\lambda) / T]=\sigma^{h}(\lambda) / \sigma(\lambda)$, and $\exp [\psi(\Lambda) / T]$ $=\tau^{h}(\Lambda) / \tau(\Lambda)$. Here $\rho(k)\left[\rho^{h}(k)\right], \sigma(\lambda)\left[\sigma^{h}(\lambda)\right]$, and $\tau(\Lambda)$ $\left[\tau^{h}(\Lambda)\right]$ are particle densities (hole densities) in $k$ space, $\lambda$ space, and $\Lambda$ space, determined by the Bethe ansatz equations (6) in the thermodynamic limit [71]. The superscript denotes the negative part of the dressed energies, which correspond to the occupied states. The positive part of the dressed energies correspond to the unoccupied states. The pressure of the system can be obtained from

$$
P_{0}=\frac{1}{2 \pi} \int_{-Q}^{Q} \epsilon^{-}(k) d k .
$$

The dressed energy equations (34)-(36) provide an elegant way to analyze the ground-state properties in terms of external fields. For $H=0$, we see that $\epsilon(k)$ has finite Fermi points. However, $\phi(\lambda)$ and $\psi(\Lambda)$ have Fermi surfaces at infinity. After taking Fourier transforms in Eqs. (35) and (36) we obtain the single dressed energy equation

$$
\epsilon(k)=k^{2}-\mu-\mu_{b}+\frac{1}{2 \pi} \int_{-Q}^{Q} \frac{2 c \epsilon^{-}(k)}{c^{2}+(\lambda-k)^{2}},
$$

which is equivalent to the dressed energy equation for the spinless Bose gas $[43,72]$ with the chemical potential $\mu_{B}$ $=\mu+\mu_{b}$. Therefore, the ground state for the mixture with $H$ $=0$ is exactly the same as that for Lieb-Lininger bosons. For strong coupling, the ground-state energy

$$
\frac{E}{L} \approx \frac{1}{3} n^{3} \pi^{2}\left(1-\frac{4}{\gamma}+\frac{12}{\gamma^{2}}\right)
$$

can be derived from Eq. (38). We have thus shown that the ground state at $H=0$ is populated only by bosons.

As the external field is turned on, the Fermi surface for $\phi(\lambda)$ rises gradually. In this case, we have

$$
\begin{gathered}
\epsilon(k)=k^{2}-\mu-\frac{1}{2} H+\frac{1}{2 \pi} \int_{-B}^{B} \frac{c \phi^{-}(\lambda)}{\frac{c^{2}}{4}+(k-\lambda)^{2}}, \\
\phi(\lambda)=-\mu_{b}+\frac{1}{2} H+\frac{1}{2 \pi} \int_{-Q}^{Q} \frac{c \epsilon^{-}(k)}{\frac{c^{2}}{4}+(\lambda-k)^{2}} .
\end{gathered}
$$

Analysis of these dressed energy equations reveal that pure Zeeman splitting between the two-component fermions does not energetically favor the fermions with spin down. Therefore, the pure Zeeman field $H$ can only drive the system from the pure boson phase into a mixture of fermions with spin up and bosons or a phase of fully polarized fermions for a large enough external field. The mixture of partially polarized fermions and bosons requires two Zeeman splitting parameters which can maintain both fermions with spin up and spin down in certain regimes (see, e.g., the various regimes for 1D three-component interacting fermions [73]). As the magnetic field is increased, more fermions with spin up populate the ground state. Beyond a critical field value, the system will be entirely occupied by fermions of one species. A phase transition is expected to occur when the field exceeds the critical value $H_{c}^{M}$. When $H \geqslant H_{c}^{M}, \phi(\lambda)$ will become nonnegative. From the dressed energy equations (40) and (41), we have

$$
\phi(\lambda) \approx-\mu_{b}+\frac{1}{2} H-\frac{P_{0} c}{c^{2} / 4+\lambda^{2}} .
$$

Now when $H=H_{c}^{M}$, the Fermi sea for the spin pseudomomenta vanishes, i.e., $\phi(0)=0$ and the density of bosons $m_{b}$ $=M_{b} / L=0$. Hence, the critical value is $H_{c}^{M}=8 P_{0} / c$, where $P_{0}$ 
is the pressure per unit length of the system, which will be determined below.

For the mixture of fermions and bosons, the critical field is twice the value of that for spin- $\frac{1}{2}$ fermions with a repulsive interaction discussed in the last section. The reason follows from the difference in their statistics. For the repulsive fermions, the ground state is antiferromagnetic, with an equal number of spin-up and spin-down fermions. As the external magnetic field is increased it reaches the critical value $H_{c}^{F}$, at which half the total number of fermions with spin down are flipped, whereas in the mixture of polarized fermions and bosons the ground state is populated only by bosons. When the field exceeds the critical value $H_{c}^{M}$, all of the bosons are driven out of the ground state while all of the fermions are polarized. Therefore, full polarization in the mixture costs twice the energy of fully polarizing the spin- $\frac{1}{2}$ fermions.

The dressed energy equations (40) and (41) can be dealt with analytically for an external field $0 \leqslant H \leqslant H_{c}$ and in the strong coupling limit $\gamma \gg 1$. Substitution of Eq. (41) into Eq. (40) and some lengthy iteration calculations with the relations $\epsilon(Q)=0$ and $\phi(B)=0$ gives the leading behavior of the pressure per unit length as

$$
\begin{aligned}
P_{0} \approx & \frac{2 \Omega^{3 / 2}}{3 \pi}+\frac{H \Omega^{1 / 2}}{2 \pi}\left[1-\frac{2}{\pi} \tan ^{-1}\left(\frac{2 B}{c}\right)\right] \\
& +\frac{8 \Omega^{2}}{3 \pi^{3} c} \tan ^{-1}\left(\frac{2 B}{c}\right)+\frac{16 B \Omega^{2}}{3 \pi^{3}\left(c^{2}+4 B^{2}\right)},
\end{aligned}
$$

where we have adopted the notation

$$
\Omega=\mu+\frac{2 \mu_{b}}{\pi} \tan ^{-1}\left(\frac{2 B}{c}\right) .
$$

In the above equations the integration boundary for $\phi(\lambda)$ is

$$
B \approx \frac{c}{2} \sqrt{\frac{8 P_{0} / c-H+2 \mu_{b}}{H-2 \mu_{b}}},
$$

and the Fermi point $Q$ can be expressed as

$$
\begin{aligned}
Q \approx & \Omega^{1 / 2}\left\{1+\frac{H}{4 \Omega}\left[1-\frac{2}{\pi} \tan ^{-1}\left(\frac{2 B}{c}\right)\right]\right. \\
& \left.+\frac{2 P_{0}}{\Omega \pi c} \tan ^{-1}\left(\frac{2 B}{c}\right)+\frac{4 P_{0} B}{\pi \Omega\left(c^{2}+4 B^{2}\right)}\right\} .
\end{aligned}
$$

The density of bosons $m_{b}$ can be estimated from the pressure (43) and the relation $m_{b}=\partial P_{0} / \partial \mu_{b}$ as

$$
\frac{m_{b}}{n} \approx \frac{2}{\pi} \tan ^{-1}\left(\frac{2 B}{c}\right)
$$

Using the relations (45) and (47) we have $\mu_{b} \approx \frac{1}{2} H$ $-4 P_{0} c^{-1} \cos ^{2}\left(\frac{m_{b} \pi}{2 n}\right)$. The chemical potentials for bosons and fermions are chosen as $\mu_{B}=\mu+\mu_{b}$ and $\mu_{F}=\mu$. Furthermore, on neglecting terms of order $O\left(1 / \gamma^{2}\right)$ and using the particle density relation $n=\partial P_{0} / \partial \mu$, we obtain the pressure per unit length and the free energy in the form

$$
\begin{gathered}
P_{0} \approx \frac{2}{3} n^{3} \pi^{2}\left[1-\frac{6}{\gamma}\left(\frac{m_{b}}{n}+\frac{\sin \left(\frac{m_{b} \pi}{n}\right)}{\pi}\right)\right], \\
F \approx-\frac{\left(n-m_{b}\right)}{2} H+\frac{1}{3} \pi^{2} n^{3}\left[1-\frac{4}{\gamma}\left(\frac{m_{b}}{n}+\frac{\sin \left(\frac{m_{b} \pi}{n}\right)}{\pi}\right)\right],
\end{gathered}
$$

from which we can examine the magnetic properties of the model.

\section{Magnetic properties}

We first see that the energy per unit length $E=F+m^{z} H$ is consistent with the results obtained in Ref. [53]. Here we take $m^{z}=\left(n-m_{b}\right) / 2$. It follows that

$$
H \approx \frac{8}{3 \gamma} \pi^{2} n^{2}\left[1+\cos \left(\frac{m_{b} \pi}{n}\right)\right]
$$

The susceptibility $\chi$ follows as

$$
\chi(H)=\frac{\partial m^{z}}{\partial H}=\frac{3 \gamma}{16 \pi^{3} n \sin \left(\frac{m_{b} \pi}{n}\right)} .
$$

From the relation (50), we find $\sin \left(\frac{m_{b} \pi}{n}\right)=2\left(\frac{3 \gamma H}{16 \pi^{2} n^{2}}\right)^{1 / 2}(1$ $\left.-\frac{3 \gamma H}{16 \pi^{2} n^{2}}\right)^{1 / 2}$. As a result the susceptibility is given by

$$
\chi \approx \frac{n}{2 \pi} \frac{1}{H^{1 / 2}\left(H_{c}^{M}-H\right)^{1 / 2}},
$$

where the critical field $H_{c}^{M} \approx \frac{16}{\gamma} E_{F}$. It is clear to see that the susceptibility is divergent in the vicinities of $H=0$ and $H$ $=H_{c}^{M}$. The magnetization in the vicinity of these points belongs to the universality class of square-root field-dependent magnetization. This indicates a van Hove type singularity of the empty band. It is evident that pure Zeeman splitting for the mixture of polarized fermions and bosons may trigger three phases: a pure boson phase when the external field is absent; a fully polarized phase when the external field exceeds the critical value $H_{c}^{M}$; and a coexisting phase of fully polarized fermions and bosons for $0<H<H_{c}^{M}$, as shown in Fig. 2.

This phase diagram is reminiscent of that of $1 \mathrm{D}$ weakly attractive fermions with population imbalance $[30,74]$, where the fully paired phase occurs only for the external field $H$ $=0$; the fermions are fully polarized for $H \geqslant H_{c}=n^{2}\left(\pi^{2}\right.$ $+2|\gamma|)$; and the paired and unpaired fermions coexist for 0 $<H<H_{c}$. However, there are subtle differences with the phase diagram for 1D strongly attractive fermions with population imbalance [21], where the bound pairs in the homogeneous system form a singlet ground state when the external field $H<H_{c 1}$. A completely ferromagnetic phase without pairing occurs when the external field $H>H_{c 2}$ and the paired and unpaired atoms coexist for an intermediate field $H_{c 1}<H<H_{c 2}$. The essential differences between the magnetic properties of the interacting Bose-Fermi mixture and 


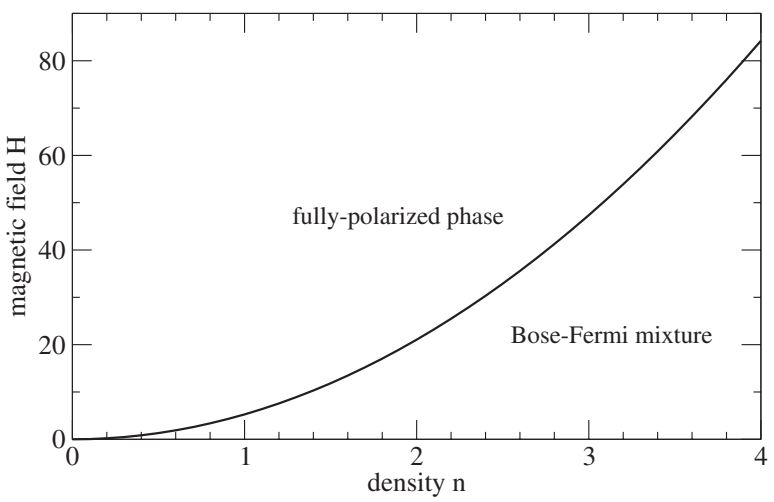

FIG. 2. Phase diagram for the 1D mixture of polarized fermions and bosons with strong coupling $c=10$.

interacting fermions with polarization are due to their different statistical signatures.

Plots of the magnetization and susceptibility versus the external magnetic field $H$ are shown in Figs. 3 and 4. The susceptibility is infinitely divergent in the vicinities of $H=0$ and $H=H_{c}^{M}$. We can also see that as the interaction strength $\gamma$ increases the critical field tends to zero. If the interaction strength is weaker, the particles have more freedom to move along the line. Consequently, there will be a strong spin fluctuation which makes it harder to fully polarize the entire system. On the other hand, for strong repulsion the spins are "frozen," thus making it easier to fully polarize the system.

For the weak coupling regime, we obtain the ground-state energy of the mixture of fully polarized fermions and bosons from the Bethe ansatz equations (2), with result [54]

$$
\frac{E}{L} \approx\left[\frac{\pi^{2} M_{1}^{3}}{3 L^{3}}+\frac{M_{b}^{2} c}{L^{2}}+\frac{2 M_{b} M_{1} c}{L^{2}}\right] .
$$

The magnetization is determined by $H \approx 8 \pi^{2}\left(\mathrm{~m}^{z}\right)^{2}-8 \mathrm{~cm}^{z}$ for $\gamma \ll 1$. These results show that in the weak coupling limit, free fermion behavior emerges for finite external field. The critical field $H_{c}^{M} \approx 2 n^{2}\left(\pi^{2}-2 \gamma\right)$ is also twice the value of that for spin- $\frac{1}{2}$ fermions with a weak interaction discussed in the

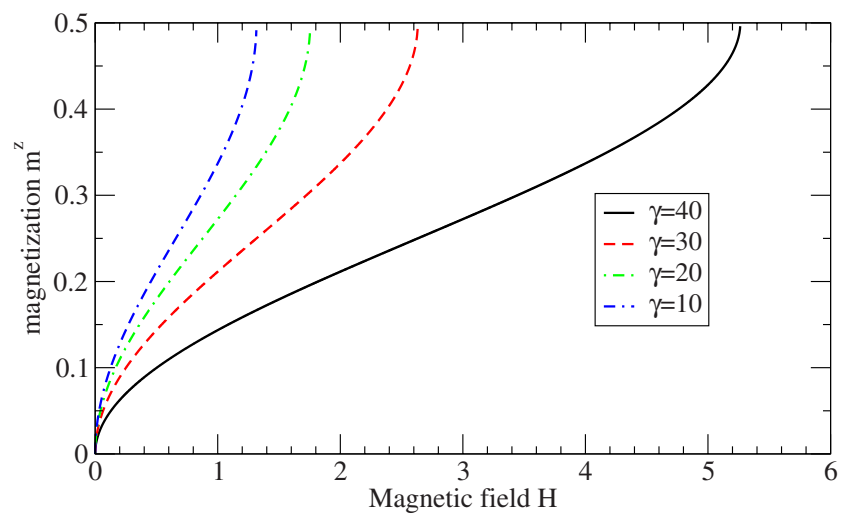

FIG. 3. (Color online) Magnetization $m^{z}(H)$ vs external field $H$ for the Bose-Fermi mixture with $n=1$ and different values of $\gamma$. The magnetization curves are plotted from Eq. (50).

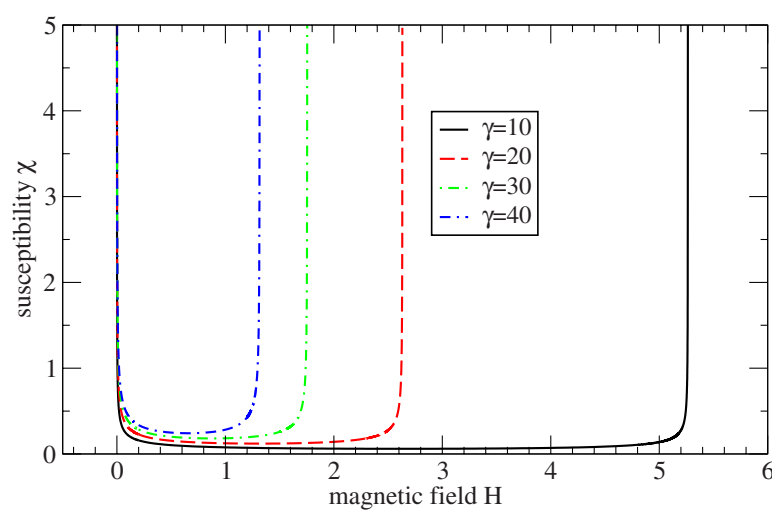

FIG. 4. (Color online) Susceptibility $\chi(H)$ vs external field $H$ for the Bose-Fermi mixture with $n=1$ and different values of $\gamma$. The susceptibility is evaluated from Eq. (51). Infinite divergencies in susceptibility are found at $H=0$ and $H=H_{c}^{M}$.

last section. This is different from the antiferromagnetic behavior of the 1D Fermi gas with population imbalance.

\section{CONCLUSION}

We have studied external field-dependent magnetic properties and statistical effects in 1D polarized strongly repulsive fermions and in a $1 \mathrm{D}$ mixture of polarized fermions and bosons with a repulsive interaction. We have found that the linear field-dependent behavior of the magnetization in the polarized Fermi gas persists for weak and finitely strong coupling regimes. The susceptibility is found to be finite. However, in the extreme limit $\gamma \rightarrow \infty$, the susceptibility is infinitely divergent due to its free spin signature. The spin-spin interaction is effectively described by the isotropic Heisenberg spin chain with antiferromagnetic coupling constant $J$ $\approx-4 E_{F} / \gamma$. A quantum phase transition from the partially polarized phase into the fully polarized phase occurs when the external field is greater than the critical value $H_{c}^{F} \approx 8 E_{F} / \gamma$ (recall Fig. 1). For the weak coupling regime, the critical field is $H_{c}^{F} \approx n^{2}\left(\pi^{2}-2 \gamma\right)$. From these configurations, we can predict the subtle segments for the 1D Fermi gas in a harmonic trapping potential: partially polarized fermions lie in the center of the cloud whereas fully polarized fermions sit in the two outer wings (see also Ref. [39]).

For the mixture of polarized fermions and bosons, analytical results for the ground-state energy, magnetization, and susceptibility have been obtained by solving the dressed energy equations for arbitrary $H$. We have shown that the ground state is only populated by bosons in the absence of a magnetic field. When the external field is applied, spin-up fermions populate the ground state. This leads to an infinitely divergent susceptibility. The fully polarized fermions and bosons coexist in the range $0<H<H_{c}^{M} \approx 16 E_{F} / \gamma$. Another phase transition from this partially polarized phase into the fully polarized Fermi phase takes place when the field $H$ $>H_{c}^{M}$ (recall Fig. 2). The susceptibility diverges. This phase diagram is somewhat reminiscent of weakly interacting attractive fermions [30], where weakly interacting BCS pairs can be viewed as strongly repulsive bosons in the mixture. 
This signature was recently observed in experiment [35]. There are subtle but essential differences between the mixture and the attractive fermions due to the different statistical signatures of the boson and the bosonic dimer. The mixture in a harmonic trapping potential has distinct segments: a boson-fermion mixed phase in the center of the cloud and a fully polarized fermion phase in the two outer wings (see also Ref. [53]). These exotic magnetic properties may also possibly be observed in experiment with ultracold fermionic and bosonic atoms through photoemission spectroscopy techniques [75].

The fundamental Fermi-Fermi and Bose-Fermi models considered here with repulsive interaction are tunable manybody systems exhibiting novel critical behavior. In these systems, the low energy physics can be described by Luttinger liquids [45]. The long distance behavior of correlation functions can be predicted from conformal field theory [52]. In general, the phenomenological Luttinger parameters seem difficult to obtain in terms of the dynamical interaction strength. However, our analytic results for the ground-state energies derived from the Bethe ansatz solution give a direct way to determine the Luttinger parameters [45]. The Luttinger parameters characterize a power law decay in correlation functions which can possibly be measured in experi- ment, such as done recently for weakly coupled spin ladders in a magnetic field [76]. This gives an opportunity to explore Luttinger liquid physics via two component interacting fermions and the Bose-Fermi mixture. Moreover, the control of effective spin-spin interaction between ultracold atoms provides a promising setting to explore critical scaling, phase transition universality classes, and entanglement in 1D many-body physics [45].

Finally, we would like to stress that while it is most convenient to use the external field $H$ in analyzing the critical behavior of the Fermi-Fermi and Bose-Fermi models, "polarization" is more widely used in cold atomic physics. However, our explicit relations between the magnetization $m^{z}$ and the external field $H$, for example, results (21), (26), and (50), set up direct links between $H$ and the polarization, which we hope will be helpful in experimental realization of these models.

\section{ACKNOWLEDGMENTS}

This work has been supported by the Australian Research Council. The authors thank J.-P. Cao, S. Chen, and H.-Q. Zhou for helpful discussions.
[1] H. Moritz, T. Stöferle, M. Köhl, and T. Esslinger, Phys. Rev. Lett. 91, 250402 (2003).

[2] B. Laburthe Tolra, K. M. O’Hara, J. H. Huckans, W. D. Phillips, S. L. Rolston, and J. V. Porto, Phys. Rev. Lett. 92, 190401 (2004).

[3] B. Paredes, A. Widera, V. Murg, O. Mandel, S. Folling, I. Cirac, G. V. Shlyapnikov, T. W. Hansch, and I. Bloch, Nature (London) 429, 277 (2004).

[4] T. Kinoshita, T. Wenger, and D. S. Weiss, Science 305, 1125 (2004); Phys. Rev. Lett. 95, 190406 (2005).

[5] H. Moritz, T. Stoferle, K. Guenter, M. Kohl, and T. Esslinger, Phys. Rev. Lett. 94, 210401 (2005).

[6] M. A. Cazalilla, J. Phys. B 37, S1 (2004); S. Giorgini, L. P. Pitaevskii, and S. Stringari, e-print arXiv:0706.3360, Rev. Mod. Phys. (to be published); V. A. Yurovsky, M. Olshanii, and D. S. Weiss, Adv. At., Mol., Opt. Phys. 55, 61 (2008).

[7] D. M. Gangardt and G. V. Shlyapnikov, Phys. Rev. Lett. 90, 010401 (2003); K. V. Kheruntsyan, D. M. Gangardt, P. D. Drummond, and G. V. Shlyapnikov, ibid. 91, 040403 (2003).

[8] M. T. Batchelor, X.-W. Guan, N. Oelkers, and C. Lee, J. Phys. A 38, 7787 (2005); Y. Hao, Y. Zhang, J. Q. Liang, and S. Chen, Phys. Rev. A 73, 063617 (2006); Y. Hao, Y. Zhang, and S. Chen, ibid. 76, 063601 (2007); S. V. Mousavi, A. del Campo, I. Lizuain, and J. G. Muga, ibid. 76, 033607 (2007).

[9] H. Buljan, R. Pezer, and T. Gasenzer, Phys. Rev. Lett. 100, 080406 (2008); F. Deuretzbacher, K. Fredenhagen, D. Becker, K. Bongs, K. Sengstock, and D. Pfannkuche, ibid. 100, 160405 (2008).

[10] Y.-Q. Li, S.-J. Gu, Z.-J. Ying, and U. Eckern, Europhys. Lett. 61, 368 (2003); J. Cao, Y. Jiang, and Y. Wang, ibid. 79, 30005 (2007).
[11] J. Links, H.-Q. Zhou, R. H. McKenzie, and M. D. Gould, J. Phys. A 36, R63 (2003); H.-Q. Zhou, J. Links, R. H. McKenzie, and X. W. Guan, ibid. 36, L113 (2003); A. Foerster and E. Ragoucy, Nucl. Phys. B 777, 831 (2007).

[12] M. B. Zvonarev, V. V. Cheianov, and T. Giamarchi, Phys. Rev. Lett. 99, 240404 (2007).

[13] S. Zöllner, H.-D. Meyer, and P. Schmelcher, Phys. Rev. Lett. 100, 040401 (2008).

[14] G. E. Astrakharchik, D. Blume, S. Giorgini, and L. P. Pitaevskii, Phys. Rev. Lett. 93, 050402 (2004).

[15] J. N. Fuchs, A. Recati, and W. Zwerger, Phys. Rev. Lett. 93, 090408 (2004).

[16] I. V. Tokatly, Phys. Rev. Lett. 93, 090405 (2004).

[17] M. T. Batchelor, M. Bortz, X. W. Guan, and N. Oelkers, J. Phys.: Conf. Ser. 42, 5 (2006); N. Oelkers, M. T. Batchelor, M. Bortz, and X. W. Guan, J. Phys. A 39, 1073 (2006).

[18] T. Iida and M. Wadati, J. Phys. Soc. Jpn. 74, 1724 (2005).

[19] G. Orso, Phys. Rev. Lett. 98, 070402 (2007).

[20] H. Hu, X.-J. Liu, and P. D. Drummond, Phys. Rev. Lett. 98, 070403 (2007).

[21] X.-W. Guan, M. T. Batchelor, C. Lee, and M. Bortz, Phys. Rev. B 76, 085120 (2007).

[22] A. E. Feiguin and F. Heidrich-Meisner, Phys. Rev. B 76, 220508(R) (2007).

[23] T. Iida and M. Wadati, J. Stat. Mech.: Theory Exp. (2007) P06011; J. Phys. Soc. Jpn. 77, 024006 (2008).

[24] M. Tezuka and M. Ueda, Phys. Rev. Lett. 100, 110403 (2008).

[25] G. G. Batrouni, M. H. Huntley, V. G. Rousseau, and R. T. Scalettar, Phys. Rev. Lett. 100, 116405 (2008).

[26] M. M. Parish, S. K. Baur, E. J. Mueller, and D. A. Huse, Phys. Rev. Lett. 99, 250403 (2007). 
[27] Z.-J. Ying, M. Cuoco, C. Noce, and H.-Q. Zhou, Phys. Rev. Lett. 100, 140406 (2008).

[28] M. Rizzi, M. Polini, M. A. Cazalilla, M. R. Bakhtiari, M. P. Tosi, and R. Fazio, Phys. Rev. B 77, 245105 (2008).

[29] M. Colomé-Tatché, G. V. Shlyapnikov, and A. M. Tsvelik, e-print arXiv:0712.4081.

[30] E. Zhao and W. Vincent Liu, e-print arXiv:0804.4461.

[31] M. Casula, D. M. Ceperley, and E. J. Mueller, e-print arXiv:0806.1747.

[32] M. W. Zwierlein, A. Schirotzek, C. H. Schunck, and W. Ketterle, Science 311, 492 (2006); M. W. Zwierlein, C. H. Schunck, A. Schirotzek, and W. Ketterle, Nature (London) 442, 54 (2006); Y. Shin, M. W. Zwierlein, C. H. Schunck, A. Schirotzek, and W. Ketterle, Phys. Rev. Lett. 97, 030401 (2006).

[33] G. B. Partridge, W. H. Li, R. I. Kamar, Y. A. Liao, and R. G. Hulet, Science 311, 503 (2006); G. B. Partridge, W. Li, Y. A. Liao, R. G. Hulet, M. Haque, and H. T. C. Stoof, Phys. Rev. Lett. 97, 190407 (2006).

[34] C. H. Schunck, Y. Shin, A. Schirotzek, M. W. Zwierlein, and W. Ketterle, Science 316, 867 (2007).

[35] Y. Shin, A. Schirotzek, C. H. Schunck, and W. Ketterle, e-print arXiv:0805.0623.

[36] A. Recati, J. N. Fuchs, and W. Zwerger, Phys. Rev. A 71, 033630 (2005).

[37] J. J. Zirbel, K.-K. Ni, S. Ospelkaus, J. P. D’Incao, C. E. Wieman, J. Ye, and D. S. Jin, Phys. Rev. Lett. 100, 143201 (2008); M. Taglieber, A.-C. Voigt, T. Aoki, T. W. Hänsch, and K. Dieckmann, ibid. 100, 010401 (2008).

[38] C. A. Stan, M. W. Zwierlein, C. H. Schunck, S. M. F. Raupach, and W. Ketterle, Phys. Rev. Lett. 93, 143001 (2004); Z. Hadzibabic, C. A. Stan, K. Dieckmann, S. Gupta, M. W. Zwierlein, A. Görlitz, and W. Ketterle, ibid. 88, 160401 (2002).

[39] M. Colomé-Tatché, e-print arXiv:0801.2640.

[40] M. Snoek, I. Titvinidze, C. Toke, K. Byczuk, and W. Hofstetter, e-print arXiv:0802.3211.

[41] J. N. Fuchs, D. M. Gangardt, T. Keilmann, and G. V. Shlyapnikov, Phys. Rev. Lett. 95, 150402 (2005).

[42] M. T. Batchelor, M. Bortz, X.-W. Guan, and N. Oelkers, J. Stat. Mech.: Theory Exp. (2006) P03016.

[43] X. W. Guan, M. T. Batchelor, and M. Takahashi, Phys. Rev. A 76, 043617 (2007).

[44] K. A. Matveev and A. Furusaki, e-print arXiv:0808.0681.

[45] T. Giamarchi, Quantum Physics in One Dimension (Oxford University Press, Oxford, 2004).

[46] D. B. M. Dickerscheid, D. van Oosten, E. J. Tillema, and H. T. C. Stoof, Phys. Rev. Lett. 94, 230404 (2005).

[47] A. Albus, F. Illuminati, and J. Eisert, Phys. Rev. A 68, 023606
(2003).

[48] L. Mathey, D.-W. Wang, W. Hofstetter, M. D. Lukin, and E. Demler, Phys. Rev. Lett. 93, 120404 (2004).

[49] L. Mathey, Phys. Rev. B 75, 144510 (2007).

[50] A. Zujev, A. Baldwin, R. T. Scalettar, V. G. Rousseau, P. J. H. Denteneer, and M. Rigol, e-print arXiv:0711.4635.

[51] M. A. Cazalilla and A. F. Ho, Phys. Rev. Lett. 91, 150403 (2003); K. K. Das, ibid. 90, 170403 (2003).

[52] H. Frahm and G. Palacios, Phys. Rev. A 72, 061604(R) (2005)

[53] A. Imambekov and E. Demler, Phys. Rev. A 73, 021602(R) (2006); Ann. Phys. (N.Y.) 321, 2390 (2006).

[54] M. T. Batchelor, M. Bortz, X. W. Guan, and N. Oelkers, Phys. Rev. A 72, 061603(R) (2005).

[55] M. Lewenstein, L. Santos, M. A. Baranov, and H. Fehrmann, Phys. Rev. Lett. 92, 050401 (2004); D. Solenov and D. Mozyrsky, ibid. 100, 150402 (2008).

[56] B. Sutherland, Phys. Rev. Lett. 20, 98 (1968).

[57] C. K. Lai and C. N. Yang, Phys. Rev. A 3, 393 (1971).

[58] E. H. Lieb and W. Liniger, Phys. Rev. 130, 1605 (1963).

[59] C. N. Yang, Phys. Rev. Lett. 19, 1312 (1967).

[60] M. Gaudin, Phys. Lett. 24, 55 (1967).

[61] E. Lieb and D. Mattis, Phys. Rev. 125, 164 (1962).

[62] M. Bortz (private communication).

[63] C. K. Lai, Phys. Rev. A 8, 2567 (1973).

[64] M. Takahashi, Thermodynamics of One-Dimensional Solvable Models (Cambridge University Press, Cambridge, 1999).

[65] F. H. L. Essler, H. Frahm, F. Göhmann, A. Klümper, and V. E. Korepin, The One-Dimensional Hubbard Model (Cambridge University Press, Cambridge, 2005).

[66] P. Schlottmann, Int. J. Mod. Phys. B 11, 355 (1997).

[67] M. T. Batchelor, X.-W. Guan, N. Oelkers, and Z. Tsuboi, Adv. Phys. 56, 465 (2007).

[68] Jen-Yee Lee, Honors thesis, Australian National University (2007).

[69] X. Du, L. Luo, B. Clancy, and J. E. Thomas, e-print arXiv:0805.1036.

[70] Z. X. Hu, Q. L. Zhang, and Y. Q. Li, J. Phys. A 39, 351 (2006).

[71] C. K. Lai, J. Math. Phys. 15, 954 (1974).

[72] C. N. Yang and C. P. Yang, J. Math. Phys. 10, 1115 (1969).

[73] X.-W. Guan, M. T. Batchelor, C. Lee, and H. Q. Zhou, Phys. Rev. Lett. 100, 200401 (2008).

[74] J. S. He, A. Foerster, X. W. Guan, and M. T. Batchelor (unpublished).

[75] J. T. Stewart, J. P. Gaebler, and D. S. Jin, e-print arXiv:0805.0026.

[76] M. Klanjsek et al., e-print arXiv:0804.2639. 